\title{
Chest CT-Derived Muscle Metrics for Sarcopenia: Choosing the Right Target
}

\author{
Palmi Shah, MD, and Mark Supanich, PhD
}

Department of Diagnostic Radiology and Nuclear Medicine, Rush University Medical Center, Chicago, IL

Sarcopenia, broadly defined as loss of skeletal muscle mass and function, often is clinically silent but evident on imaging. Shen et al. ${ }^{1}$ in 2004 showed that total skeletal muscle mass on a single computed tomography (CT) section at the L3 level is closely related to lean body mass. This finding led to the establishment of validated genderbased cutoff values for defining sarcopenia. Currently, CT is considered the gold standard for body composition analysis $^{2}$ of cancer patients because of its ability to derive muscle metrics from staging or follow-up CT scans without the need to re-radiate the patient.

In oncology, body composition assessment is most commonly performed by CT quantification of skeletal muscle mass and density at the L3 level. It is of prognostic value for several GI cancers ${ }^{3}$ and also is associated with postoperative complications, increased mortality, chemotherapy toxicity, and overall survival in several cancers. Recently it has been suggested that CT-based low muscle attenuation, an indirect indicator of muscle quality, may be a more sensitive indicator of sarcopenia. ${ }^{4}$

Clinically, lung cancer patients have the highest prevalence of cancer cachexia, ${ }^{5}$ but their routine imaging workup does not always include the L3 vertebral body level. The lack of standardized chest CT-derived metrics for sarcopenia has challenged the use of this important imaging biomarker in research studies. A literature search shows significantly heterogeneous use of total skeletal

(C) Society of Surgical Oncology 2021

First Received: 20 October 2021

Accepted: 21 October 2021;

Published Online: 13 November 2021

P. Shah, MD

e-mail: Palmi_Shah@Rush.edu muscle mass at different levels such as the L1, T4, and T5 levels, the erector spinae muscle, and pectoralis muscle metrics without validation or well-researched cutoff levels.

In their recent analysis, Grønberg et al. ${ }^{6}$ compared the L3-derived total muscle area to the T4 level and showed poor correlation between these two levels. Other authors also have shown that arm position (raised vs by the side) can alter the value of the total muscle area obtained on the single-slice CT scan. ${ }^{7}$ In addition, CT slice thickness, algorithms, radiation dose, and other factors can alter muscle metrics. ${ }^{8}$ A metanalysis by Yang et al. ${ }^{3}$ researching the predictive value of sarcopenia in lung cancer showed that in these studies, scan levels and cutoff values were variable. The ability to leverage muscle metrics on chest CT scans depends on finding the optimal target to help define sarcopenia.

The pectoralis muscle area assessed on a single-slice CT scan appears to correlate well with a fat-free mass measured by dual-energy X-ray absorptiometry (DXA). ${ }^{9}$ In addition, the CT-derived pectoralis muscle area was predictive of respiratory dysfunction for patients with chronic obstructive pulmonary disease (COPD). ${ }^{9}$ In this issue of Annals of Surgical Oncology, Sun et al. ${ }^{10}$ in their report have uniquely combined both the normalized pectoralis muscle area (pectoralis muscle area/body mass index) and mean muscle density to generate a unique integrative pectoralis muscle index (IPMI) to prognosticate postsurgical complications and the overall survival impact on patients with stages I to IIIA non-small cell lung cancer (NSCLC). This innovative approach combines the two CTderived metrics that define sarcopenia: muscle mass and quality. In addition, by using respiratory muscle-imaging biomarkers, the authors indirectly incorporate the key metric used for preoperative assessment: pulmonary function tests. As others have done, the authors divided the patients into four sex-specific quartiles given the lack of 
standardized cutoff IPMI values. Stage I NSCLC patients in the lowest quartile also exhibited the worst 5-year overall survival $(P=0.004)$, and the lowest quartile also was an independent adverse prognostic risk factor for overall survival.

Use of the body mass index (BMI) for normalization of the pectoralis muscle area is unusual because most muscle metrics are normalized using patient height in meters. Therefore, comparing the use of height with the BMI for muscle metric normalization needs to be researched. Since this was a single-institution retrospective analysis, the pectoralis muscle index needs further investigation and validation with large-population studies to define its role in risk stratification. Specifically, age-, gender-, and racebased metrics need to be established and validated before its use in research and clinical practice.

Current evidence shows that imaging muscle biomarkers can help prognosticate outcomes for oncology patients, but no studies have established the association between sarcopenia and progression-free survival. To date, no established interventions are available to limit sarcopenia progression, although several nutritional, pharmacologic, and rehabilitative measures currently under investigation are considered of value. Validated CT-derived L3 muscle metrics have yet to be incorporated into clinical practice because their derivation currently is tedious and semiautomated.

Conceptually, IMPI could be the first step in the right direction as we search for more reliable, reproducible, and quantifiable imaging biomarkers on chest CT scan. Maybe the future availability of fully automated software with machine learning incorporated into the regular clinical workflow will help screen and identify high-risk patients for personalization of their management to obtain the most optimal outcome. Routine chest CT thoracic muscle analysis to refine risk stratification models for lung cancer patients may be the future.

\section{DISCLOSURES}

There are no conflicts of interest.

\section{REFERENCES}

1. Shen W, Punyanitya M, Wang Z, et al. Total body skeletal muscle and adipose tissue volumes: estimation from a single abdominal cross-sectional image. J Appl Physiol. 2004;97:2333-8.

2. Prado CM, Heymsfield SB. Lean tissue imaging: a new era for nutritional assessment and intervention. JPEN J Parenter Enteral Nutritr. 2014;38:940-53.

3. Yang M, Shen Y, Tan L, et al. Prognostic value of sarcopenia in lung cancer: a systematic review and meta-analysis. Chest. 2019;156:101-11.

4. Graffy PM, Liu J, Pickhardt PJ, et al. Deep learning-based muscle segmentation and quantification at abdominal CT: application to a longitudinal adult screening cohort for sarcopenia assessment. Br J Radiol. 2019;92:20190327.

5. Baracos VE, Reiman T, Mourtzakis M, et al. Body composition in patients with non-small cell lung cancer: a contemporary view of cancer cachexia with the use of computed tomography image analysis. Am J Clin Nutr. 2010;91:1133S-S1137.

6. Grønberg BH, Sjøblom B, Wentzel-Larsen T, et al. A comparison of CT-based measures of skeletal muscle mass and density from the Th4 and L3 levels in patients with advanced non-small cell lung cancer. Eur J Clin Nutr. 2019;73:1069-76.

7. van Heusden HC, Swartz JE, Chargi N, et al. Feasibility of assessment of skeletal muscle mass on a single cross-sectional image at the level of the fourth thoracic vertebra. Eur J Radiol. 2021;142:109879.

8. Fuchs G, Chretien YR, Mario J, et al. Quantifying the effect of slice thickness, intravenous contrast, and tube current on muscle segmentation: implications for body composition analysis. Eur Radiol. 2018;28:2455-63.

9. McDonald ML, Diaz AA, Ross JC, et al. Quantitative computed tomography measures of pectoralis muscle area and disease severity in chronic obstructive pulmonary disease: a cross-sectional study. Ann Am Thorac Soc. 2014;11:326-34.

10. Sun C, Anraku M, Kawahara T, et al. Combination of skeletal muscle mass and density predicts postoperative complications and survival of patients with non-small cell lung cancer. Ann Surg Oncol. 2021. https://doi.org/10.1007/10434.1534-4681

Publisher's Note Springer Nature remains neutral with regard to jurisdictional claims in published maps and institutional affiliations. 